\title{
Impacts of $A$ Taste of African Heritage: A Culinary Heritage Cooking Course
}

\author{
Marla Reicks, $\mathrm{PhD}, \mathrm{RDN}^{1}$; Abby Gold, PhD, $\mathrm{MPH}, \mathrm{RD}^{2}$; Nicole Tran ${ }^{1}$; \\ Kelly LeBlanc, MLA, RD, $\mathrm{LDN}^{3}$
}

\begin{abstract}
Objective: To examine changes in cooking and dietary behaviors and health outcomes following participation in A Taste of African Heritage, a culinary heritage cooking course.

Design: One group, pre-post program design, surveys, and physical assessments.

Setting: Community centers, churches, health care settings, homes, and housing complexes.

Participants: Participants $(n=586,84 \%$ women) were recruited by partner organizations from 2013 -2018 across 21 states and the District of Columbia.

Intervention(s): A 6-week cooking curriculum that teaches history, nutrition, and cooking techniques to reconnect participants with the vibrant, healthy traditions of the African Diaspora.

Main Outcome Measure(s): Weekly frequency of cooking, food group intake, and exercise assessed by surveys, measured weight, waist circumference, and blood pressure.

Analysis: Linear and logistic mixed-effects models with random intercepts for participant and teacher, sex and site type as covariates comparing pre-post physical measurements and binary behavioral outcomes, $P<$ 0.0033 for statistical significance after Bonferroni correction.

Results: Intake frequencies of fruit, vegetable, and greens and exercise frequency were improved from preprogram to postprogram (all $P<0.0021$ ). Weight, waist circumference, and systolic blood pressure were also improved from preprogram to postprogram (all $P<0.0001)$.

Conclusions and Implications: Participation in A Taste of African Heritage was associated with positive behavior changes and health outcomes. Integrating cultural heritage and behaviors are positive components to connect participants to healthy old ways or traditions.
\end{abstract}

Key Words: cooking curriculum, African culinary heritage, adults, eating patterns, weight (J Nutr Educ Behav. 2022;54:388-396.)

Accepted November 18, 2021. Published online February 10, 2022.

\section{INTRODUCTION}

The African Heritage Diet is described by Oldways, ${ }^{1}$ a nonprofit organization focused on improving health through adopting the old ways of shared cultural tradition, as a "way of eating based on the healthy food traditions of people with African roots." Adverse social circumstances challenge the retention of healthy traditional eating patterns from Africa to the Americas. ${ }^{2}$ The African Heritage Diet is based on traditions of the African Diaspora, which includes the American South, West and Central Africa, the Caribbean, and parts of South America. The A Taste of African Heritage (ATOAH) program, developed by Oldways, is a 6-week cooking and nutrition curriculum for adults that teaches culinary history, nutrition, and cooking techniques on the basis of the African Heritage Diet.

\footnotetext{
${ }^{1}$ Department of Food Science and Nutrition, University of Minnesota, Department of Food Science and Nutrition, St Paul, MN

${ }^{2}$ Center for Family Development, University of Minnesota Extension, St Paul, MN

${ }^{3}$ Oldways, Boston, MA

Conflict of Interest Disclosure: The authors have not stated any conflicts of interest.

Address for correspondence: Marla Reicks, Department of Food Science and Nutrition, University of Minnesota, 1334 Eckles Ave, St Paul, MN 55108; E-mail: mreicks@umn.edu

(C) 2021 The Authors. Published by Elsevier Inc. on behalf of Society for Nutrition Education and Behavior. This is an open access article under the CC BY-NC-ND license (http:// creativecommons.org/licenses/by-nc-nd/4.0/)

https://doi.org/10.1016/j.jneb.2021.11.008
}

The program is intended to reconnect participants with the vibrant, healthy traditions of the African Diaspora and promote eating patterns on the basis of plant foods, including leafy greens, whole grains, beans, and tubers.

Eating patterns and diet quality are associated with lower risk of dietrelated chronic disease, impaired cognitive function, and lower total and cause-specific mortality in African American men and women. ${ }^{3-8}$ Key factors mediating risk were waist circumference and body mass index. ${ }^{6,8}$ Several studies have shown that diet quality among African American adults on the basis of national or regional samples was indicative of not meeting national dietary recommendations. ${ }^{9,10}$ Higher empty calorie intake and lower consumption of fruit, dairy, and total protein foods than a national sample were observed among African American adults in the southeastern US. ${ }^{9}$ 
National Health and Nutrition Examination (NHANES) data (2003-2012) also showed that foreign-born African American adults had higher diet quality scores than US-born counterparts for vegetables, fruit, and percentage of whole grains, ${ }^{11}$ indicating that nativity may be an important consideration for interventions for the diverse US African American population.

Data from national samples of US adults (NHANES 2007-2008 and NHANES 2007-2010) showed that more than half of non-Hispanic Black adults in the study had low or medium dinner cooking frequency, ${ }^{12}$ and that African American race was significantly associated with being more likely to never to cook dinner at home. ${ }^{13}$ When dinner cooking frequency was high, more fiber and less fat and sugar were consumed. ${ }^{2}$ Among non-Hispanic Black adults (NHANES 2007-2010), diet quality, including higher dinner vegetable and lower empty calorie intake, was associated with higher cooking frequency. ${ }^{14}$ Home meal preparation was also positively associated with positive beliefs and self-efficacy toward healthy foods among a sample of African American adults from urban, low-income areas. ${ }^{15}$

Cooking skills can help equip people to make healthier food choices. ${ }^{16}$ Several nutrition intervention programs have incorporated a culinary component that has helped participants transform nutrition knowledge into action with positive diet and health outcomes. ${ }^{17,18}$ Kong et a ${ }^{19}$ reported that 17 out of 28 interventions that included culturally adapted strategies showed improvements in diet and weight outcomes among African American women. Commonly used strategies were those that reflected a group's values and drew from their experiences. An 8-week intervention study that made culturally sensitive modifications to a diet program led to improvements in blood pressure (BP), weight, and other health parameters among African American participants. ${ }^{20}$ A 2week intervention in which African American adults were fed a traditional, high-fiber, low-fat Africanstyle diet resulted in reduced colon inflammation and proliferation biomarkers of cancer risk. ${ }^{21} \mathrm{~A}$ switch to a high-fat, low-fiber Western-type diet among a group of rural South Africans resulted in reversed changes in many parameters. ${ }^{21}$ Haslam et $\mathrm{al}^{22}$ found lower odds of colon cancer in Black men with better Mediterranean diet scores than White men with higher Mediterranean diet scores. Additional behavioral and clinical evidence is needed to demonstrate the effectiveness of culturally relevant culinary programming, particularly among African Americans.

The current study used evaluation data collected before and after participation in the ATOAH program from a convenience sample of adults from 2013-2018. The goal of the program is to improve cooking skills and frequency and eating habits on the basis of plant foods, including leafy greens, whole grains, beans, and tubers, and thereby address health issues of African Americans who are at greater risk for heart disease, diabetes, and obesity than other population groups. ${ }^{23}$ The program harnesses 2 powerful channels, culture and community, to create a supportive social environment and positive weekly routine that connects and motivates students along their journey. Unlike other, more impersonal diet and health programs, the heritage-based approach could be a strong motivator for change. Classes bring to light a culinary legacy and often-unsung cultural ownership of healthy eating for people of African descent through hands-on experience.

The purpose of this study was to evaluate changes in health and dietary behavior outcomes from preprogram to post-ATOAH program in a subset of adults participating in the program across the US from 2013 -2018. Findings may inform decisions about culinary programming from a cultural perspective for adults with an African heritage.

\section{METHODS}

\section{Research Design}

This study was based on a 1-group, pre-post program design to evaluate the diet and health impacts of the ATOAH program.

\section{Participants}

Oldways conducted the ATOAH program among 1,823 adults from 2013 -2018. Participants were recruited through Oldways partner organizations serving as hosts and/or instructors using email marketing, flyers, and word-of-mouth referrals. Host organizations were in 21 US states and the District of Columbia, primarily in the eastern and southern US. Data from a subset of the 1,823 participants who completed the program from 2013-2018 were included in the data analysis for this evaluation study. Inclusion criteria were participants who had data for some or all of the health impact measures (BP, weight or waist circumference) and had data for behavioral survey questions $(n=586)$. The data analysis for the current study was not considered human subject research by the University of Minnesota as defined by US Department of Health and Human Services and Food and Drug Administration regulations. ${ }^{24,25}$

\section{Procedures}

Program development. A Taste of African Heritage was developed by Oldways with a grant from the Walmart Foundation. First, Oldways assembled an advisory committee of nutrition scientists, culinary historians, and experts ${ }^{26}$ in African diasporic health and history to develop the African Heritage Diet Pyramid, which was used as the framework for creating the curriculum. The curriculum includes 6 weekly lessons (1.75 -2 hours per lesson), each addressing a specific food group from the African Heritage Diet Pyramid with 4 components: (1) an introduction, (2) an historical and nutritional discussion, (3) a cooking lesson (2-3 recipes per lesson), and (4) a time to eat together and reflect (Supplementary Table 1). The curriculum applies selected constructs of the transtheoretical model ${ }^{27}$ (stages of change) and social cognitive theory ${ }^{26}$ (self-efficacy, behavioral capability, and observational learning) ${ }^{28}$ (Supplementary Figure 1). Participants sign a Healthy Heritage Pledge in the first lesson to move into the contemplation stage. Building cooking and 
shopping skills and receiving recipes, grocery lists, and supplementary resources help participants through the preparation stage and into the action stage. Program graduates are encouraged to transition into the maintenance stage by participating in Oldways' social media groups or teaching a class series of their own. Social Cognitive Theory ${ }^{28}$ constructs applied include self-efficacy and behavioral capability through acquisition of shopping and cooking skills. The group nature of the classes also serves as a form of observational learning. After review by experts for nutrition, cultural and culinary content, the curriculum was pilot-tested at 15 sites across the country to refine the flow of the lessons and optimize the recipes before being formally implemented in 2012.

Program implementation. Oldways staff began recruiting partners to teach and host ATOAH by networking, attending conferences and cultural festivals, and engaging in active phone and email outreach to community centers, health centers, and cultural organizations. Since then, ATOAH has grown in a grassroots fashion. Interested teachers approach Oldways to learn more about the class series. To ensure program fidelity across sites, instructor training involves a 1-hour teacher training webinar from Oldways, a quiz about the principles of the African Heritage Diet, and a phone call with an Oldways staff member to discuss logistics and answer questions about best practices for teaching. Teachers may include registered nurses, registered dietitian nutritionists, chefs, and other health professionals. The Teacher's Manual describes best practices for instruction, including how to arrange the classroom (grocery lists and required tools), an agenda with timing for each activity or topic, and a detailed curriculum script.

\section{Measures and Data Collection Procedures}

Health impacts. Systolic blood pressure (SBP) and diastolic blood pressure (DBP) were measured once in $\mathrm{mmHg}$ immediately before and after the program for each participant using a digital blood pressure monitor and cuff provided by Oldways (Omron 5 Series, Omron Healthcare, Inc) along with printed instructions for measuring and recording $\mathrm{BP}^{29}$ to maintain consistency across classes. If the program was held in a health care facility, available BP measuring equipment was used. Blood pressure was classified into the high blood pressure (HBP) stage according to the Seventh Report of the Joint National Committee on the Prevention, Detection, Evaluation, and Treatment of High Blood Pressure (2004-2017). ${ }^{29}$ Blood pressure stages were defined as (1) normal BP (SBP, < 120; DBP, < $80 \mathrm{mmHg}$ ), (2) prehypertension (SBP, 120-139 mmHg; DBP, 80-89 mmHg), (3) hypertension, stage 1 (SBP, 140-159; DBP, 90-99 mmHg), and (4) hypertension, stage 2 (SBP, $\geq$ $160 \mathrm{mmHg}$; DBP $\geq 100 \mathrm{mmHg}$ ).

Weight was measured in pounds once for each participant before and after the program with digital bathroom scales provided by Oldways with printed instructions ${ }^{30}$ for measuring and recording the weight to maintain consistency across classes. If the class was held in a health care facility, available weighing equipment was used.

Waist circumference was measured in inches once for each participant before and after the program with coated nonstretchy measuring tapes provided by Oldways with printed instructions to measure the waist in inches at a horizontal line 1in above the umbilicus. ${ }^{30}$ Teachers recorded participant sex (female or male) based on visual evaluation when physical measurements were completed at baseline.

Diet, exercise, and cooking frequency. Similar to other community-based cooking interventions, such as Cooking Matters ${ }^{31}$ and the Expanded Food and Nutrition Education program, ${ }^{32}$ a low respondent burden, preintervention and postintervention behavioral survey was administered to evaluate effectiveness. The survey was developed by the curriculum development advisory committee and included in the 15-site pilot. The frequency of intake of foods from various food groups was assessed before and after the program with questions including "How many times a week do you eat (greens, whole grains, beans, tubers, vegetables, fruits, vegetarianbased meals)?" with response options of (1) never, (2) 1-2 times a week, (3) 3-4 times a week, (4) 5-6 times a week, and (5) every day. Questions also addressed exercise (How often do you exercise a week, including walking?), cooking at home (How many times a week do you cook at home?), and using herbs and spices (How many times a week do you cook with herbs and spices?) with the same response options.

Postprogram questions about program outcomes. Participants were asked to respond in writing to several openended questions postprogram about the program and outcomes, including (1) suggested curriculum changes, (2) how to describe African heritage foods to someone unfamiliar with these foods, (3) what surprised participants about the class, and (4) which recipes were most interesting, which recipes they prepared at home and reasons for not preparing recipes. Another question asked, "Are history and heritage positive motivators for living and eating well?" with yes/no response options. An additional 2 questions examined in-depth included (1) Has this program changed the way you eat at all? If so, how? and (2) What was the biggest obstacle getting in the way of cooking and eating healthy? These questions were selected for indepth analysis because they were most directly related to overall behavior change and its success, and therefore useful for verifying quantitative findings. A codebook was developed by the first author (M.R.), an experienced qualitative researcher, and an undergraduate nutrition student trained by the first author (N.T.) in coding and constant comparative qualitative analysis methods. ${ }^{33,34}$ Investigators (M.R. and N.T.) examined responses from the first 100 participants together for both questions. The remaining responses were then coded by 1 researcher and verified by the other researcher. Discussion about any disagreement in assigning codes was followed by reassignment of codes after consensus was reached. Finally, the 2 researchers summarized results by organizing codes into higher-order themes and reporting themes with representative quotes. 


\section{Statistical Analyses}

Statistical software (SAS, version 9.4; SAS Institute Inc, 2013) was used for the analyses. Descriptive statistics (means and standard deviations, frequencies, and percentages) were used to describe participant and program characteristics and health and behavioral outcomes. Linear mixed-effects models with random intercepts for participant and teacher and sex and site type as covariates were used to assess changes between preprogram and postprogram for health outcomes (weight, waist circumference, and BP). For behavioral outcomes, responses were collapsed into 2 categories: $\leq$ 4 times per week (responses of never, 1 -2 times per week, and 3-4 times per week) and $\geq 5$ times per week (5 -6 times per week and every day) similar to other studies. ${ }^{13,31}$ Logistic mixed-effects models with random intercepts for participant and teacher and sex and site type as covariates were used for binary behavioral outcomes (frequency of food group intake, exercise, cooking, and use of herbs and spices). A Bonferroni adjustment was used for multiple tests for changes in outcomes to decrease the probability of finding significant effects by chance. All statistical tests were then conducted at the 0.0033 $(0.05 / 15)$ significance level.

\section{RESULTS}

Of the 586 total participants who met the inclusion criteria for this evaluation study, the majority were female $(\mathrm{n}=488, \quad 83.7 \%)$. Classes $(\mathrm{n}=99)$ which included the subset of participants who met the inclusion criteria, consisted of 6 weekly lessons which were conducted across 21 states and the District of Columbia (1-16 classes per state/District of Columbia) from 2013 to 2018 (Supplementary Table 2). In some cases, data were used from the entire class, whereas for other cases, data were only used from some participants in the class. A mean of 6 participants who met the inclusion criteria for this evaluation study were in each class (1-34 participants) with 38 to 165 participants per year. Sixty-seven different teachers taught the classes. Participants attended classes in 1 out of 4 types of settings: churches $(n=181$ participants, $30.9 \%)$; community venues including community centers, senior centers, schools, libraries and farmers' markets $(n=210,35.8 \%)$; health care facilities $(\mathrm{n}=90,15.4 \%)$; and homes and housing complexes $(\mathrm{n}=105,17.9 \%)$.

The number of participants with $\mathrm{BP}$, waist, and weight measures varied by class, so the overall number of participants for these values varied (Table 1). Based on preprogram measures, mean $\mathrm{BP}$ values were indicative of prehypertension. Preprogram to postprogram differences in BP, waist, and weight measures were adjusted for the effect of sex and site type. Mean SBP was lower from preprogram to postprogram $(P<0.0001)$. The HBP stage was significantly lower from preprogram to postprogram ( $P$ $<0.0001)$. Mean weight in pounds was lower postprogram than preprogram $(P<0.0001)$. Mean preprogram waist circumference values were above the optimal BMI-specific waist circumference thresholds for estimating cardiometabolic risk in African American women and men with normal weight, overweight, or obesity. ${ }^{35}$ After the program, mean waist circumference was lower compared with preprogram $(P<0.0001)$.

Significant positive changes in intake of greens, fruits, and vegetables and exercise were observed after the program compared with before the program (Table 2) according to logistic mixed-effects model analyses adjusted for the effect of sex and site type. For example, participants had 1.51 times the odds of eating greens at least 5-6 times per week postprogram than preprogram $(P=0.0021)$. Participants had 1.82 times the odds of eating vegetables at least $5-6$ times per week postprogram than preprogram $(P<0.0001), 1.56$ times the odds of eating fruit at least 5-6 times per week postprogram than preprogram $(P=0.0006)$, and 1.81 times the odds of exercising at least 5-6 times per week postprogram than prepro$\operatorname{gram}(P<0.0001)$. Cooking frequency and frequency of using herbs and spices were not significantly different postprogram than preprogram $(P=0.03$ and $P=0.02$, respectively). Almost all (98\%) participants indicated that history and heritage were positive motivators for living and eating well.

In response to the open-ended question about behavior change because of program participation, 3 general themes were identified on the basis of (1) changes in purchasing

Table 1. Changes in Blood Pressure, Waist Circumference, and Weight From Preprogram to Postprogram

\begin{tabular}{|c|c|c|c|c|c|c|c|}
\hline Measure & $\mathbf{n}$ & $\begin{array}{l}\text { Preprogram } \\
\text { Mean (SD) }\end{array}$ & $\begin{array}{c}\text { Postprogram } \\
\text { Mean (SD) }\end{array}$ & $\begin{array}{l}\text { Preprogram to } \\
\text { Postprogram } \\
\text { Proportion } \\
\text { Improved }\end{array}$ & $\begin{array}{c}\text { Effect } \\
\text { Estimate }\end{array}$ & $\begin{array}{l}\text { Confidence } \\
\text { Limits }\end{array}$ & $P^{a}$ \\
\hline Systolic BP, mmHG & 511 & $136.7(19.4)$ & $132.3(19.0)$ & 60.3 & -4.33 & -5.77 to -2.89 & $<.0001$ \\
\hline Diastolic BP, mmHG & 511 & $83.5(13.3)$ & $82.0(13.9)$ & 53.8 & -1.44 & -2.63 to -0.24 & 0.0184 \\
\hline HBP stage & 511 & $3.1(1.1)$ & $2.9(1.1)$ & 29.7 & -0.19 & -0.28 to -0.09 & $<.0001$ \\
\hline Waist circumference, in & 475 & $40.4(6.9)$ & $39.8(6.9)$ & 54.1 & -0.61 & -0.77 to -0.46 & $<.0001$ \\
\hline Weight, lb & 561 & $191.9(51.2)$ & $190.5(51.3)$ & 59.7 & -1.35 & -1.68 to -1.02 & $<.0001$ \\
\hline
\end{tabular}

BP indicates blood pressure; HBP, high blood pressure stage.

${ }^{a} P$ for linear mixed models with random intercepts for participant and teacher and sex and site type as covariates, Bonferroni correction for multiple comparisons (0.05/15 comparisons, $P<0.0033)$, and means and SDs are unadjusted sample statistics; ${ }^{\text {bHBP stage }}{ }^{29}$ : 1 = normal; 2 = prehypertension; 3 = hypertension, stage 1 ; and $4=$ hypertension, stage 2. 
Table 2. Changes in Frequency of Food Group Intake and Other Behaviors From Preprogram to Postprogram

\begin{tabular}{|c|c|c|c|c|}
\hline Food Group & $\begin{array}{c}\text { Postprogram } \\
\leq 4 \text { Times/Wk, } n(\%)^{a}\end{array}$ & $\begin{array}{c}\text { Postprogram } \\
\geq 5 \text { Times/Wk, n (\%) }\end{array}$ & $\begin{array}{c}\text { Odds Ratio }^{\text {b }} \\
\text { (Confidence Interval) }^{\text {(Confe }}\end{array}$ & $P^{c}$ \\
\hline \multicolumn{5}{|l|}{ Greens } \\
\hline Preprogram $\leq 4$ times/wk & $309(55.0)$ & $94(16.7)$ & $1.51(1.16-1.96)$ & 0.0021 \\
\hline Preprogram $\geq 5$ times/wk & $48(8.5)$ & $111(19.8)$ & & \\
\hline \multicolumn{5}{|l|}{ Whole grains } \\
\hline Preprogram $\leq 4$ times/wk & 359 (63.9) & $72(12.8)$ & $1.21(0.91-1.60)$ & 0.1925 \\
\hline Preprogram $\geq 5$ times/wk & $54(9.6)$ & $77(13.7)$ & & \\
\hline \multicolumn{5}{|l|}{ Beans } \\
\hline Preprogram $\leq 4$ times/wk & $472(86.8)$ & $34(6.3)$ & $1.65(1.05-2.59)$ & 0.0299 \\
\hline Preprogram $\geq 5$ times/wk & $16(2.9)$ & $22(4.0)$ & & \\
\hline \multicolumn{5}{|l|}{ Tubers } \\
\hline Preprogram $\leq 4$ times/wk & $471(87.1)$ & $37(6.8)$ & $1.61(1.02-2.55)$ & 0.0421 \\
\hline Preprogram $\geq 5$ times/wk & $18(3.3)$ & $15(2.8)$ & & \\
\hline \multicolumn{5}{|l|}{ Vegetables } \\
\hline Preprogram $\leq 4$ times/wk & $181(32.1)$ & $101(17.9)$ & $1.82(1.41-2.35)$ & $<0.0001$ \\
\hline Preprogram $\geq 5$ times/wk & $30(5.3)$ & $252(44.7)$ & & \\
\hline \multicolumn{5}{|l|}{ Fruit } \\
\hline Preprogram $\leq 4$ times/wk & $223(39.1)$ & $95(16.7)$ & $1.56(1.21-2.01)$ & 0.0006 \\
\hline Preprogram $\geq 5$ times/wk & $42(7.4)$ & $210(36.8)$ & & \\
\hline \multicolumn{5}{|l|}{ Vegetarian-based meals } \\
\hline Preprogram $\leq 4$ times/wk & $450(83.0)$ & $42(7.8)$ & $1.77(1.18-2.65)$ & 0.0056 \\
\hline Preprogram $\geq 5$ times/wk & $15(2.8)$ & $35(6.5)$ & & \\
\hline \multicolumn{5}{|l|}{ Exercise } \\
\hline Preprogram $\leq 4$ times/wk & $365(66.0)$ & 75 (13.6) & $1.81(1.35-2.44)$ & $<0.0001$ \\
\hline Preprogram $\geq 5$ times/wk & $22(4.0)$ & $91(16.5)$ & & \\
\hline \multicolumn{5}{|l|}{ Cooking frequency } \\
\hline Preprogram $\leq 4$ times/wk & $303(53.6)$ & $63(11.2)$ & $1.35(1.03-1.77)$ & 0.0287 \\
\hline Preprogram $\geq 5$ times/wk & $43(5.7)$ & $167(29.6)$ & & \\
\hline \multicolumn{5}{|l|}{ Use of herbs and spices } \\
\hline Preprogram $\leq 4$ times/wk & $296(53.8)$ & 73 (13.3) & $1.38(1.05-1.81)$ & 0.0201 \\
\hline Preprogram $\geq 5$ times/wk & $41(7.5)$ & $140(25.5)$ & & \\
\hline
\end{tabular}

${ }^{\mathrm{a}}$ Frequencies and percentages are based on the unadjusted sample.; ${ }^{\mathrm{b}}$ Odd ratios are interpreted as participants having $\mathrm{n}$ times the odds of eating food groups at least 5-6 times per wk postprogram compared to preprogram; ${ }^{\mathrm{c}} P$ for logistic mixed-effects models with random intercepts for participant and teacher, and sex and site type as covariates. For behavioral outcomes, responses were collapsed into 2 categories: $\leq 4$ times per wk (responses of never, $1-2$ times per wk, and 3-4 times per wk) and $\geq$ 5 times per wk (5-6 times per wk and every d). Bonferroni correction for multiple comparisons $(0.05 / 15$ comparisons, $P=0.0033)$

("Yes, I'm more careful when shopping to check the label and ingredients"), (2) changes in preparation and trying new foods ("Added quinoa to my diet! Also cook greens differently"), and (3) changes in the frequency of using recipes ("I'm more open now to trying and enjoying African dishes" and "It has given me confidence to try more traditional foods"). A theme was also identified regarding specific changes in foodrelated behaviors that addressed eating more greens and vegetables with subthemes including (1) enjoying meatless meals ("I'm inspired to make more meals without meat," "I have more greens on my plate than meat and potatoes," and "I incorporate more vegetables as a main meal/ foundation"), (2) improving vegetable cooking methods ("Steaming, eating raw veggies, not too much frying" and "I am mindful of eating and preparing my greens in a healthier manner") and (3) eating a greater variety of vegetables. A theme regarding changes in using herbs and spices for seasoning food was related to using more herbs and spices with subthemes, including (1) using them instead of salt ("Less salt but more flavor! More herbs and spices"), and (2) using a variety " Adding herbs and spices to my food that I would not have before."

In addition to themes regarding behavior changes, a theme was identified that included change in awareness and knowledge from program participation ("Absolutely, much more aware of my food choices, preparation and the nutritional value of what I'm eating" and "Yes, I have so many more options for good nutrition"). In response to the question about obstacles to cooking and eating healthy, commonly mentioned obstacles were time, food cost, not enjoying cooking, lack of experience or knowledge, lack of energy/ motivation, preferring other foods including "junk food" and sweets, consideration of family members' preferences, and for some, having to cook for 1 and eating alone.

\section{DISCUSSION}

Overall, preprogram vs postprogram evaluation survey data showed that 
participation in the ATOAH program improved dietary behaviors, indicating a shift toward a more plant-based diet. Health outcomes including systolic BP, HBP stage, weight, and waist circumference were also improved; however, improvements may still not have reduced levels to meet standards for optimum health. ${ }^{29,35}$ For example, average weight loss from preprogram to postprogram was 1.38 pounds compared with other studies that reported a greater magnitude of postintervention weight loss (2-5 kg after 6 months $^{19}$ or 14.8 pounds after 8 weeks ${ }^{20}$ ). Differences in health outcomes such as total weight loss could be accounted for by the amount of time spent with participants, level of personalized attention, and overall program objectives. Future studies to establish efficacy should be completed with a stronger study design on the basis of the inclusion of a control group and longer-term follow-up.

Positive health outcomes included weight loss, lowered SBP, and decreased waist circumference. Because fruit and vegetable intake increased in the current study, an association may exist between an increased fruit and vegetable intake and the positive changes observed in anthropometric variables. Schwingshackl et $\mathrm{al}^{36}$ also observed changes in fruit and vegetable intake with subsequent changes in anthropometric variables. Participants reported eating more fruits, vegetables, and greens in the current study, representing a potential overall shift to a plantbased diet and the likelihood of improving diet quality scores. A previous study had indicated that Healthy Eating Index (HEI) 2005 scores on the basis of NHANES data (2003-2004) were $56 \%, 54 \%$, and $22 \%$ of the maximum scores for total fruit, total vegetables, and dark green and orange vegetables and legumes, respectively among non-Hispanic African American adults. ${ }^{10}$ Nutrition education may generate dietary behavior modification through skills to identify and shop for healthful food items. ${ }^{37}$ Previous culturally relevant nutrition education was associated with greater program satisfaction and dietary patterns for African American women. ${ }^{38-40}$ The ATOAH curriculum integrated culturally relevant messages focusing on traditional foods, and building connections to African heritage.

Cooking frequency was not significantly different in the current study when examined in terms of odds of cooking at home at least 5-6 times per week postprogram than preprogram with $11.2 \%$ indicating a low frequency before the program and a high frequency after the program. Farmer et $\mathrm{al}^{14}$ showed that an increase from 0-1 times/wk of cooking dinner at home to 2-5 times/wk among non-Hispanic Black adults was associated with an increase in total HEI score from 44.0 to 46.8 $(P=0.023)$ and total vegetable component HEI score from 2.41 to 2.56 $(P=0.003)$. These findings may indicate that overall dietary quality and alignment of vegetable intake with the 2020-2025 Dietary Guidelines for Americans, ${ }^{41}$ were improved for $11.2 \%$ of the participants in the current study with increased cooking frequency. However, these findings may also indicate that identified barriers in response to the open-ended question about obstacles to cooking more often may have inhibited greater changes, such as time, food cost, and not enjoying cooking. Similar barriers to home cooking are identified in other qualitative studies. ${ }^{42,43}$ In addition, changes in food group intake may have resulted from adding or substituting those foods to meals regularly cooked at home, rather than increasing cooking frequency.

Several factors may explain why males comprised only about $16 \%$ of the total participants in the current study. A review of the effectiveness of cooking programs showed that these programs have largely enrolled women, with men underrepresented as participants. ${ }^{17,18}$ Boundaries of home, work, and leisure commonly associated with domestic gender roles may differ for some men and women, ${ }^{44}$ in line with the percentages of non-Hispanic Black men and women who were reported to cook (37\% vs 63\%, respectively) in 2016 on the basis of nationally representative time-use data. ${ }^{45}$ Cooking enjoyment may also be a factor, Hartmann et $\mathrm{al}^{16}$ found enjoyment was a predictor of cooking skills, especially for men and that women had higher cooking skills at all ages than men. Changing eating behaviors in pursuit of health may pose a threat to normative masculinity for some men, as it challenges men to "admit vulnerability and relinquish power" over their bodies. ${ }^{46}$ Because ATOAH outcomes benefit males and females, efforts should be made to increase ATOAH participation among males, possibly by providing classes that specifically recruit and enroll males.

In the current study, the frequency of whole-grain intake before the program was low, consistent with a report based on the NHANES (2013 -2016) for non-Hispanic Black participants indicating that only about $14 \%$ of total grains intake was consumed as whole grains vs the recommended $50 \%$ of total grains as whole grains. ${ }^{47}$ Whole-grain intake frequency did not significantly improve during the ATOAH program. The ATOAH curriculum included whole grain recipes throughout the 6 lessons that focused on common whole-grain foods such as brown rice and ancient grains such as millet, sorghum, quinoa, and teff. ${ }^{48,49}$ However, availability and cost of ancient grains may limit use because some must be imported vs being grown domestically. A narrative review of barriers to whole grain consumption among children and adults indicated that the main factors influencing consumption were cost, taste, difficulty to identify, and lack of knowledge and availability. ${ }^{50}$ In particular, the availability of supermarkets, which may limit the availability of quality foods, was found to be low across Black census tracts in the US. ${ }^{51}$

A compelling finding of the current study was that almost all (98\%) participants indicated that history and heritage were positive motivators for living and eating well, consistent with some participants indicating the use of traditional foods and African dishes in response to a question about changes they attributed to program participation. Research suggests that successful nutrition interventions in African American communities should include culturally palatable foods regularly enjoyed in Black cuisine. ${ }^{52}$ In addition, the 2020-2025 Dietary Guidelines for 
Americans $^{38}$ emphasize the importance of incorporating cultural food traditions. However, the role of heritage as a direct motivator for health is under-explored in the literature. Cultural identity is shown to shape food enjoyment, ${ }^{53}$ suggesting when programs highlight African American cultural identity, the desire to eat healthy African heritage foods is enhanced. This suggestion is consistent with the positive changes in food group intake frequency observed in the current study.

Designed as a community-based cooking program rather than a research study, rigorous evaluation methods were not implemented, including a control group, longerterm follow-up, assessment of intervention delivery and dose, and collection of comprehensive information about demographic characteristics. Information on nativity, race, and ethnicity was not collected, and the only information available about participant demographic characteristics for those included in the data analysis was sex. Because this study was conducted to evaluate a program, it was not designed to answer research questions or be based on power analysis in the context of multiple sites. Although the questionnaire was pilottested with participants in 15 classes, information about validity and reliability are lacking. In addition, estimating the intake frequency of beans and tubers separately from vegetables may have been difficult, making estimates of intakes less valid. As with other evaluation studies of cooking programs, ${ }^{17,18}$ selection bias is a limitation for the current study because participants interested in cooking are likely to join cooking classes resulting in a higher likelihood of identifying positive outcomes.

\section{IMPLICATIONS FOR RESEARCH AND PRACTICE}

Integrating cultural heritage, beliefs, and behaviors are essential components when the objective is to connect participants to healthy "old ways" or traditions. Many cultures are represented in the African American population, such as those of Caribbean,
South American, African nations, and regional cultures in the US. A Taste of African Heritage addresses traditions across these many cultures. As Di Noia et $\mathrm{al}^{39}$ indicated, designing culturally relevant nutrition education that includes concepts such as morality, spirituality, the centrality of women, historical legacy, and cultural meaning of food are essential to successfully improve dietary practices of African Americans. For example, a large part of the ATOAH curriculum included communal food preparation with the time taken to share meals and stories. Although developed as a nutrition education curriculum, ATOAH ultimately was a cultural experience for participants that led to healthy lifestyle changes. This approach has implications for other curriculum designed to reconnect communities to their heritage and traditions. More rigorous research on the effectiveness of the ATOAH program could include a stronger study design, such as the inclusion of a control group, longerterm followup, and recruitment strategies that address sampling biases. Research could also incorporate process evaluation measures to report lesson attendance and retention of participants for pre and postassessments.

\section{ACKNOWLEDGMENTS}

Research reported in this publication was supported by the National Center for Advancing Translational Sciences of the National Institutes of Health Award Number UL1TR002494. The content is solely the responsibility of the authors and does not necessarily represent the official views of the National Institutes of Health.

The authors thank those who participated in this program. The authors also thank Sara Baer-Sinnott, Constance Brown-Riggs, and Alissa Perteet-Jackson for their insightful comments on the manuscript and Nicholas Marka for statistical consultation.

\section{SUPPLEMENTARY DATA}

Supplementary data related to this article can be found at https://doi. org/10.1016/j.jneb.2021.11.008.

\section{REFERENCES}

1. Oldways. About us. https://oldwayspt. org/about-us . Accessed March 4, 2021.

2. Kris-Etherton PM, Petersen KS, Velarde G, et al. Barriers, opportunities, and challenges in addressing disparities in diet-related cardiovascular disease in the United States. J Am Heart Assoc. 2020;9:e014433.

3. Yu D, Sonderman J, Buchowski MS, et al. Healthy eating and risks of total and cause-specific death among lowincome populations of African-Americans and other adults in the southeastern United States: a prospective cohort study. PLoS Med. 2015;12:e1001830. discussion e1001830.

4. Wright RS, Waldstein SR, Kuczmarski MF, et al. Diet quality and cognitive function in an urban sample: findings from the Healthy Aging in Neighborhoods of Diversity across the Life Span (HANDLS) study. Public Health Nutr. 2017;20:92-101.

5. Boggs DA, Ban Y, Palmer JR, Rosenberg L. Higher diet quality is inversely associated with mortality in AfricanAmerican women. J Nutr. 2015;145: 547-554.

6. Cespedes EM, Hu FB, Tinker L, et al. Multiple healthful dietary patterns and type 2 diabetes in the Women's Health Initiative. Am J Epidemiol. 2016;183:622-633.

7. Weng LC, Steffen LM, Szklo M, Nettleton J, Chambless L, Folsom AR. A diet pattern with more dairy and nuts, but less meat is related to lower risk of developing hypertension in middleaged adults: the Atherosclerosis Risk in Communities (ARIC) Study. Nutrients. 2013;5:1719-1733.

8. Howard G, Cushman M, Moy CS, et al. Association of clinical and social factors with excess hypertension risk in Black compared with White US adults. JAMA. 2018;320:1338-1348.

9. Richards Adams IK, Figueroa W, Hatsu I, et al. An examination of demographic and psychosocial factors, barriers to healthy eating, and diet quality among African American adults. Nutrients. 2019;11:519.

10. Hiza HAB, Casavale KO, Guenther PM, Davis CA. Diet quality of Americans differs by age, sex, race/ethnicity, income, and education level. J Acad Nutr Diet. 2013;113:297-306.

11. Brown AGM, Houser RF, Mattei J, et al. Diet quality among US-born and 
foreign-born non-Hispanic blacks: NHANES 2003-2012 data. Am J Clin Nutr. 2018;107:695-706.

12. Wolfson JA, Bleich SN. Is cooking at home associated with better diet quality or weight-loss intention? Public Health Nutr. 2015;18:1397-1406.

13. Virudachalam S, Long JA, Harhay MO, Polsky DE, Feudtner C. Prevalence and patterns of cooking dinner at home in the USA: National Health and Nutrition Examination Survey (NHANES) 2007 -2008. Public Health Nutr. 2014;17: 1022-1030.

14. Farmer N, Wallen GR, Yang L, Middleton KR, Kazmi N, Powell-Wiley TM. Household cooking frequency of dinner among non-Hispanic black adults is associated with income and employment, perceived diet quality and varied objective diet quality, HEI (Healthy Eating Index): NHANES analysis 2007 -2010. Nutrients. 2019;11:2057.

15. Garcia MT, Sato PM, Trude ACB, et al. Factors associated with home meal preparation and fast-food sources use among low-income urban African American adults. Ecol Food Nutr. 2018;57:13-31.

16. Hartmann C, Dohle S, Siegrist M. Importance of cooking skills for balanced food choices. Appetite. 2013;65: 125-131.

17. Reicks M, Kocher M, Reeder J. Impact of cooking and home food preparation interventions among adults: a systematic review (2011-2016). J Nutr Educ Behav. 2018;50. 148-172e1.

18. Reicks M, Trofholz AC, Stang JS, Laska MN. Impact of cooking and home food preparation interventions among adults: outcomes and implications for future programs. J Nutr Educ Behav. 2014;46: 259-276.

19. Kong A, Tussing-Humphreys LM, Odoms-Young AM, Stolley MR, Fitzgibbon ML. Systematic review of behavioural interventions with culturally adapted strategies to improve diet and weight outcomes in African American women. Obes Rev. 2014;15 (suppl 4):62-92.

20. Ard JD, Rosati R, Oddone EZ. Culturally sensitive weight loss program produces significant reduction in weight, blood pressure, and cholesterol in eight weeks. $J$ Natl Med Assoc. 2000;92:515-523.

21. O'Keefe SJD, Li JV, Lahti L, et al. Fat, fibre and cancer risk in African Americans and rural Africans. Nat Commun. 2015;6:6342.
22. Haslam A, Robb SW, Hébert JR, Huang H, Ebell MH. Greater adherence to a Mediterranean diet is associated with lower prevalence of colorectal adenomas in men of all races. Nutr Res. 2017;48:76-84.

23. Cunningham TJ, Croft JB, Liu Y, Lu H, Eke PI, Giles WH. Vital signs: racial disparities in age-specific mortality among Blacks or African Americans United States, 1999-2015. MMWR Morb Mortal Wkly Rep. 2017;66:444456. https://www.cdc.gov/mmwr/ volumes/66/wr/mm6617e1.htm? s_cid $=$ mm6617e1_w. Accessed March 4, 2021.

24. Department of Health and Human Services, Office for Human Research Protections. Code of Federal Regulations, 45 CFR 46, 2018 Requirements (2018 Common Rule). https://www. hhs.gov/ohrp/regulations-and-policy/ regulations/45-cfr-46/revised-common-rule-regulatory-text/index.html. Accessed January 10, 2022.

25. U.S. Food \& Drug Administration. Regulations: Good Clinical Practice and Clinical Trials. https://www.fda. gov/science-research/clinical-trialsand-human-subject-protection/regulations-good-clinical-practice-and-clinical-trials. Accessed January 10, 2022.

26. Oldways. AH\&H Advisory Committee. https://oldwayspt.org/programs/ african-heritage-health/ahh-advisorycommittee. Accessed March 4, 2021.

27. Prochaska JO, Redding CA, Evers K. The transtheoretical model and stages of change. In: Glanz K, Rimer BK, Viswanath K, eds. Health Behavior and Health Education: Theory, Research, and Practice, 4th Ed. Jossey-Bass, Inc; 2008:97-117.

28. McAlister AL, Perry CL, Parcel GS. How individuals, environments, and health behaviors interact: social cognitive theory. In: Glanz K, Rimer BK, Viswanath K, eds. Health Behavior and Health Education: Theory, Research, and Practice, (4th Ed). Jossey-Bass, Inc; 2008:167-185.

29. National Heart Lung, and Blood Institute. The Seventh Report on the Joint National Committee on Prevention, Detection, Evaluation, and Treatment of High Blood Pressure. National Institutes of Health, U.S. Department of Health and Human Services. NIH Publ; 2004:045230. https://www.nhlbi.nih.gov/ files/docs/guidelines/jnc7full.pdf.
30. Lohman TG, Roche AF, Martorell R. Anthropometric Standardization Reference Manual. Human Kinetics Books; 1988.

31. Pinard CA, Uvena LM, Quam JB, Smith TM, Yaroch AL. Development and testing of a revised cooking matters for adults survey. Am J Health Behav. 2015;39:866-873.

32. Auld G, Baker S, Conway L, Dollahite J, Lambea MC, McGirr K. Outcome effectiveness of the widely adopted EFNEP Curriculum Eating Smart-Being Active. J Nutr Educ Behav. 2015;47:19-27.

33. Saldaña J. The Coding Manual for Qualitative Researchers. 2nd ed. Sage Publications; 2013.

34. Miles B, Huberman A, Saldaña J. Qualitative Data Analysis: A Methods Sourcebook. 3rd ed. Sage Publications; 2014.

35. Staiano AE, Bouchard C, Katzmarzyk PT. BMI-specific waist circumference thresholds to discriminate elevated cardiometabolic risk in white and African American adults. Obes Facts. 2013;6: 317-324

36. Schwingshackl L, Hoffmann G, KalleUhlmann T, Arregui M, Buijsse B, Boeing $\mathrm{H}$. Fruit and vegetable consumption and changes in anthropometric variables in adult populations: a systematic review and meta-analysis of prospective cohort studies. PLoS One. 2015; 10:e0140846.

37. Barnidge EK, Baker EA, Schootman M, Motton F, Sawicki M, Rose F. The effect of education plus access on perceived fruit and vegetable consumption in a rural African American community intervention. Health Educ Res. 2015;30: 773-785

38. Kreuter MW, Haughton LT. Integrating culture into health information for African American women. Am Behav Sci. 2006;49:794-811.

39. Di Noia J, Furst G, Park K, Byrd-Bredbenner C. Designing culturally sensitive dietary interventions for African Americans: review and recommendations. Nutr Rev. 2013;71:224-238.

40. Williams JH, Auslander WF, de Groot M, Robinson AD, Houston C, HaireJoshu D. Cultural relevancy of a diabetes prevention nutrition program for African American women. Health Promot Pract. 2006;7:56-67.

41. US Department of Agriculture. US Department of Health and Human Services. Dietary Guidelines for Americans; 2020-2025. 9th Edition USDA; 2020. 
42. Wolfson JA, Bleich SN, Smith KC, Frattaroli S. What does cooking mean to you?: perceptions of cooking and factors related to cooking behavior. Appetite. 2016;97:146-154.

43. Lavelle F, McGowan L, Spence M, et al. Barriers and facilitators to cooking from 'scratch' using basic or raw ingredients: a qualitative interview study. Appetite. 2016;107:383-391.

44. Meah A. Reconceptualizing power and gendered subjectivities in domestic cooking spaces. Prog Hum Geogr. 2014;38:671-690.

45. Taillie LS. Who's cooking? Trends in US home food preparation by gender, education, and race/ethnicity from 2003 to 2016. Nutr J. 2018;17:41.

46. Contois EJH. Diners,Dudes, and Diets: How Gender and Power Collide in Food Media and Culture. The University of North Carolina Press; 2020:16.
47. Ahluwalia N, Herrick KA, Terry AL, Hughes JP. Contribution of whole grains to total grains intake among adults aged 20 and over: United States, 2013-2016. NCHS Data Brief, 341. Hyattsville, MD: National Center for Health Statistics; 2019:1-8.

48. Cooper R. Re-discovering ancient wheat varieties as functional foods. $J$ Tradit Complement Med. 2015;5:138-143.

49. Boukid F, Folloni S, Sforza S, Vittadini E, Prandi B. Current trends in ancient grains-based foodstuffs: insights into nutritional aspects and technological applications. Compr Rev Food Sci Food Saf. 2018;17:123-136.

50. Meynier A, Chanson-Rollé A, Riou E. Main factors influencing whole grain consumption in children and adults- $\mathrm{A}$ narrative review. Nutrients. 2020;12:2217.

51. Bower KM, Thorpe RJ, Rohde C, Gaskin DJ. The intersection of neigh- borhood racial segregation, poverty, and urbanicity and its impact on food store availability in the United States. Prev Med. 2014;58:33-39.

52. Sterling SR, Bowen SA. The potential for plant-based diets to promote health among Blacks living in the United States. Nutrients. 2019;11:2915.

53. Hackel LM, Coppin G, Wohl MJA, Van Bavel JJ. From groups to grits: social identity shapes evaluations of food pleasantness. J Exp Soc Psychol. 2018;74:270-280.

\section{ORCIDs}

Marla Reicks: http://orcid.org/00000001-6726-9408

Abby Gold: http://orcid.org/0000-

0001-5966-4440

Kelly LeBlanc: http://orcid.org/00000003-0406-1583 